\section{MULTIPARAMETRIC MAPPING TO UNDERSTAND PATHOPHYSIOLOGY IN CARDIAC AMYLOIDOSIS}

${ }^{1}$ Tushar Kotecha, ${ }^{1}$ Ana Martinez-Naharro, ${ }^{2}$ Thomas A Treibel, ${ }^{1}$ Rohin Francis, ${ }^{2}$ Sabrina Nordin, ${ }^{2}$ Amna Abdel-Gadir, 'Daniel S Knight, 'Giulia Zumbo, ${ }^{2}$ Stefania Rosmini, ${ }^{2}$ Viviana Maestrini, ${ }^{2}$ Heerajnarain Bulluck, ${ }^{1}$ Roby Rakhit, ${ }^{1}$ Ashutosh D Wechalekar, ${ }^{3}$ Peter Kellman, 'Julian D Gillmore, ${ }^{2} J a m e s$ C Moon, ${ }^{1}$ Philip N Hawkins, ${ }^{1}$ Marianna Fontana. ${ }^{1}$ Royal Free Hospital, London, UK; ${ }^{2}$ Barts Heart Centre, London, UK; ${ }^{3}$ National Heart, Lung and Blood Institute, USA

\subsection{6/heartjnl-2017-311399.1}

Background The prognosis and treatment of the 2 main types of cardiac amyloidosis, immunoglobulin light chain (AL) and transthyretin (ATTR) amyloidosis are substantially influenced by cardiac involvement. ATTR amyloidosis has better prognosis than AL despite more amyloid infiltration. This paradox suggests additional mechanisms of damage in AL amyloidosis. The aim of this study was to assess the presence and prognostic significance of oedema in a large population of patients with cardiac amyloidosis and compare findings among those with TTR and AL types.

Methods and results 286 patients (100 with systemic AL amyloidosis (including 49 patients scanned prior to commencing chemotherapy), 163 with cardiac ATTR amyloidosis, 12 with suspected cardiac ATTR amyloidosis (grade 1 on ${ }^{99 m} \mathrm{Tc}-\mathrm{DPD}$ ) and 11 asymptomatic individuals with amyloidogenic transthyretin(TTR) mutations), and 30 healthy volunteers were recruited. All subjects underwent CMR with T1 and T2 mapping, and ECV quantification. Left ventricular(LV) mass and ECV were higher in ATTR compared to AL whilst LV ejection fraction $(\mathrm{EF})$ was significantly lower $(\mathrm{p}<0.001)$. There was no significant difference in ECV between treated and untreated AL (Figure 1a). T2 was increased in cardiac amyloidosis compared to healthy volunteers with the degree of elevation being the highest in untreated AL patients (T2: $56.3 \pm 4.9 \mathrm{~ms} \mathrm{AL}$ untreated, $54.0 \pm 4.5 \mathrm{~ms}$ AL treated, 54.3 $\pm 4.1 \mathrm{~ms}$ ATTR, $48.9 \pm 2.0 \mathrm{~ms}$ controls, $\mathrm{p}<0.05$ for all except treated $\mathrm{AL}$ vs ATTR) (Figure 1b). During follow up (22.8 \pm 14.7 months), 75 (28\% of AL group and $25 \%$ of ATTR group) patients died.
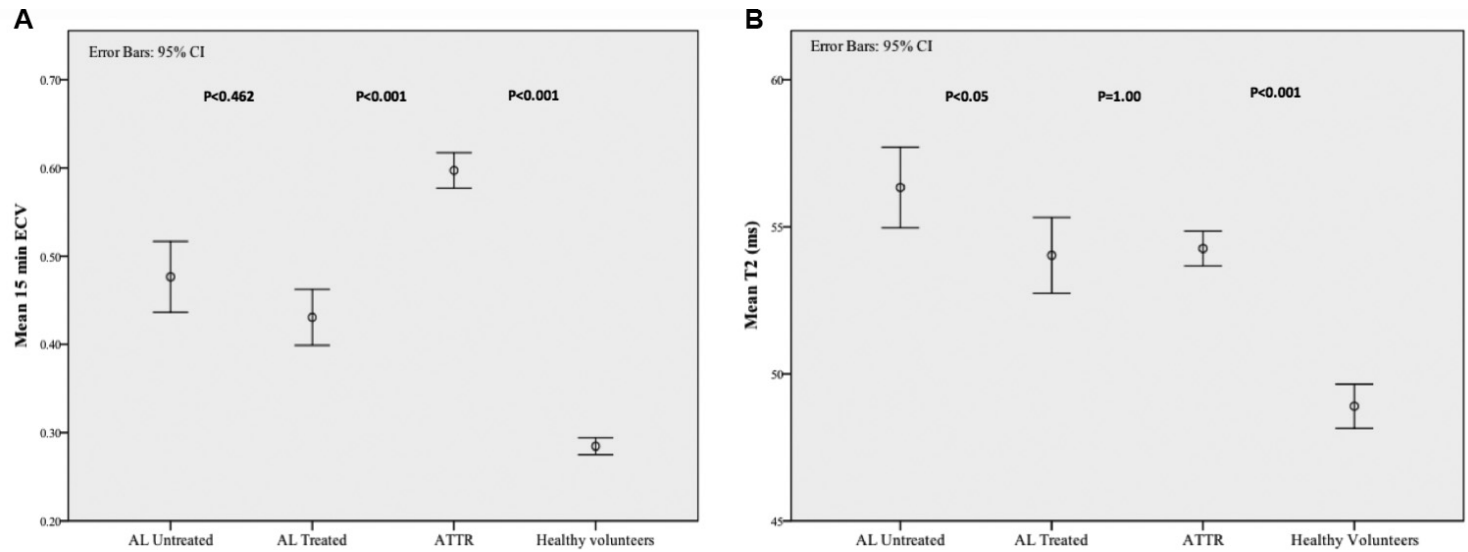

Abstract 001 Figure 1 a. Mean ECV in cardiac AL (treated and untreated patients) and ATTR amyloidosis compared to healthy volunteers. b. Mean $\mathrm{T} 2$ in cardiac AL (treated and untreated patients) and ATTR amyloidosis compared to healthy volunteers.

A

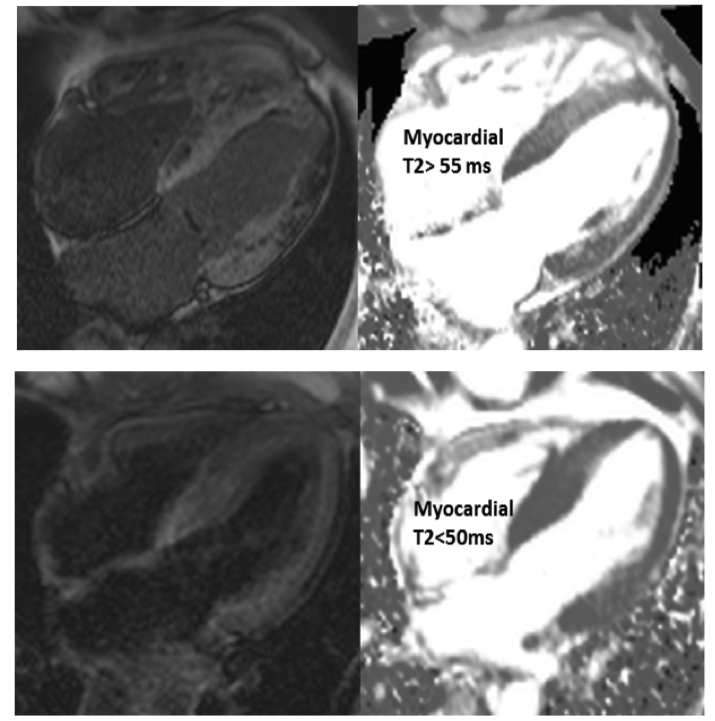

B

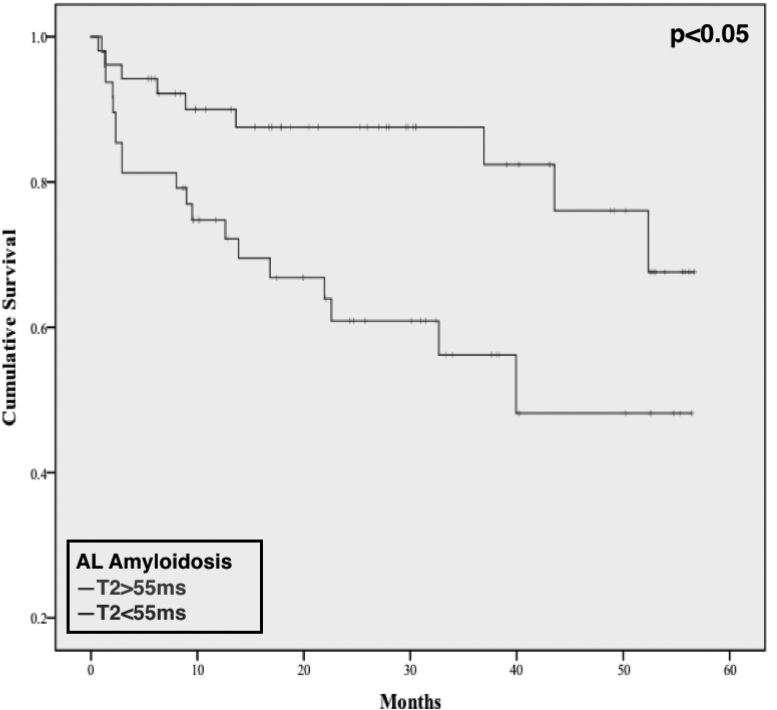

Abstract 001 Figure 2 a. Examples of two patients with AL amyloidosis and transmural late gadolinium enhancement, one with eleveted T2 (top) and the other with normal T2 (bottom). b. Kaplan-Meier survival curve of patients with AL amyloidosis with high T2 and normal T2 values. 
Using Cox regression models, T2 predicted death in AL amyloidosis (hazard ratio, HR,1.48, 95\% CI 1.20-1.82) and remained significant after adjusting for EF and ECV (HR 1.31, 95\% CI 1.04-1.66) (Abstract 1. Figure 2).

Conclusion Patients with AL amyloidosis have a worse prognosis compared to ATTR despite having less cardiac amyloid infiltration. T2 was significantly higher in untreated AL amyloidosis consistent with oedema, and was an independent predictor of prognosis. The higher ECV in ATTR was consistent with higher amyloid infiltration. These findings highlight the unique role of CMR with multiparametric mapping for characterising the cardiac effects of systemic amyloidosis and risk stratification in this population.

\section{SUDDEN CARDIAC DEATH RISK STRATIFICATION IN PATIENTS WITH MILD DILATED CARDIOMYOPATHY}

${ }^{1}$ Brian P Halliday, ${ }^{1}$ Ankur Gulati, ${ }^{1}$ Aamir Ali, ${ }^{1}$ Kaushik Guha, ${ }^{2}$ Simon Newsome, ${ }^{1}$ Monika Arzanausikaite, ${ }^{1}$ Vassilios S Vassiliou, ${ }^{1}$ Amrit Lota, ${ }^{1}$ Upasana Tayal, ${ }^{1}$ Zohya Khalique, ${ }^{1}$ Cemil Izgi, ${ }^{1}$ Francisco Alpendurada, ${ }^{1,3}$ John GF Cleland, ${ }^{1}$ Dudley J Pennell, 'Sanjay K Prasad. 'NIHR Biomedical Research Unit, Cardiovascular Magnetic Resonance Unit and Department of Cardiology, Royal Brompton Hospital, UK; ${ }^{2}$ London School of Hygiene and Tropical Medicine, UK; ${ }^{3}$ Robertson Centre for Biostatistics, University of Glasgow, UK

\subsection{6/heartjnl-2017-311399.2}

Background The DANISH trial emphasised that the selection of patients with dilated cardiomyopathy (DCM) for implantable cardioverter defibrillators (ICD) needs to be improved. Registries demonstrate that the major burden of sudden cardiac death (SCD) occurs in those with a left ventricular ejection fraction (LVEF) $>35 \%$. Those at high-risk of SCD with milder reductions in LVEF may gain greater quality-adjusted life years from successful ICD therapy compared to those with more severe reductions, due to a lower risk of death from competing non-sudden causes. Variables that identify patients with milder reductions in LVEF at high-risk of SCD are required.

Methods We prospectively investigated the utility of mid-wall late gadolinium enhancement (LGE) cardiovascular magnetic resonance (CMR) to predict SCD and aborted SCD in consecutive patients with DCM and LVEF $>40 \%$ seen in our cardiomyopathy service or referred for CMR between 2000 and 2011. Those with potential pre-existing indications for ICD implantation were excluded. The presence of LGE was determined by a specialist blinded to clinical data. A panel blinded to CMR data adjudicated end-point occurrences.

Results Of 399 patients (145 women, median age 50 years, median LVEF 50\%) followed for a median of 4.6 years, 18 of $101(17.8 \%)$ with LGE reached the pre-specified end-point, compared to 7 of 298 (2.3\%) without (HR 9.2; 95\% CI 3.9$21.8 ; \mathrm{p}<0.0001)$ (Figure 1). Nine patients (8.9\%) with LGE compared to $6(2.0 \%)$ without (HR 4.9; 95\% CI 1.8-13.5; $\mathrm{p}=0.002$ ) died suddenly, whilst 10 patients $(9.9 \%)$ with LGE compared to 1 (0.3\%) without (HR 34.8; 95\% CI 4.6-266.6; $\mathrm{p}<0.001$ ) had aborted SCD. Following adjustment based on propensity score, LGE predicted the composite end-point (HR 8.0; 95\% CI 3.3-19.5; p<0.0001), SCD (HR 4.6; 95\% CI $1.6-13.1 ; \mathrm{p}=0.005)$ and aborted SCD (HR 32.9; 95\% CI $4.3-249.9 ; \mathrm{p}<0.001)$. Estimated hazard ratios for the primary end-point for patients with a LGE extent of $0 \%-2.5 \%, 2.5 \%-$ $5 \%$ and $>5 \%$ compared to those without LGE were 10.6
(95\%CI 3.9-29.4), 4.9 (95\% CI 1.3-18.9) and 11.8 (95\% CI 4.3-32.3).

Conclusion Mid-wall LGE identifies patients with DCM and a LVEF $>40 \%$ with an 8 -fold increased risk of SCD and aborted SCD, who may benefit from ICD implantation.

Acknowledgements BPH is supported by a British Heart Foundation Clinical Research Training Fellowship. The study has also been supported by the Alexander Jansons Foundation and CORDA.

\section{PRECISE PHENOTYPING WITH CMR IDENTIFIES MODERATE ALCOHOL CONSUMPTION AS AN IMPORTANT PHENOTYPIC MODIFIER OF TITIN CARDIOMYOPATHY}

${ }^{1,2} \mathrm{U}$ Tayal, ${ }^{3} \mathrm{~S}$ Newsome, ${ }^{1} \mathrm{~N}$ Whiffin, ${ }^{2} \mathrm{R}$ Buchan, ${ }^{2} \mathrm{R}$ Walsh, ${ }^{1,2} \mathrm{PJ}$ Barton, ${ }^{1,2} \mathrm{JS}$ Ware,

${ }^{1,4}$ SA Cook, ${ }^{1,2}$ SK Prasad. 'National Heart Lung Institute, Imperial College London, UK;

${ }^{2}$ Royal Brompton Hospital, London, UK; ${ }^{3}$ Department of Medical Statistics, London School of Hygiene and Tropical Medicine, UK; ${ }^{4}$ Duke National University Hospital, Singapore

\subsection{6/heartjnl-2017-311399.3}

Background Truncating variants in titin (TTNtv) are the commonest genetic cause of dilated cardiomyopathy (DCM). They are notable for variable penetrance and expressivity, suggestive of environmental or genetic modifiers.

Purpose Undertake deep phenotyping by cardiac MRI (CMR) to evaluate alcohol and hypertension as phenotypic modifiers of TTNtv cardiomyopathy.

Methods Prospectively recruited DCM patients underwent comprehensive clinical evaluation, CMR with late-gadolinium enhancement and TTN sequencing.

Results Overall, 733 subjects, mean age 53.4 \pm 14.4 years, 476 men $(65.0 \%)$ were recruited. TTNtv were found in 82 $(11.2 \%)$ patients.

Abstract 003 Table 1 Regression analysis evaluating TTNtv and alcohol excess as predictors of LVEF

\begin{tabular}{|c|c|c|c|c|c|c|}
\hline \multirow[b]{2}{*}{ Variable } & \multicolumn{3}{|c|}{ Unadjusted analysis } & \multicolumn{3}{|c|}{ Adjusted analysis* } \\
\hline & $\begin{array}{l}\text { Estimate } \\
\text { (change } \\
\text { in LVEF, } \\
\% \text { ) }\end{array}$ & $\begin{array}{l}95 \% \\
\text { confidence } \\
\text { intervals }\end{array}$ & $P$ value & $\begin{array}{l}\text { Estimate } \\
\text { (change } \\
\text { in LVEF, } \\
\% \text { ) }\end{array}$ & $\begin{array}{l}95 \% \\
\text { confidence } \\
\text { intervals }\end{array}$ & $P$ value \\
\hline
\end{tabular}

or alcohol excess

TTNtv, no alcohol $\quad 0.06 \quad-3.0$ to $\quad 0.97 \quad-0.3 \quad-3.2$ to $\quad 0.85$

$\begin{array}{llll}\text { excess } & 3.1 & 2.7\end{array}$

Alcohol excess, no $\quad-2.1 \quad-4.7$ to $\quad 0.12 \quad-0.6 \quad-3.2$ to $\quad 0.61$

$\begin{array}{lll}\text { TTNtv } & 0.5 & 1.9\end{array}$

TTNtv and alcohol $\quad-11.8 \quad-18.6$ to $\quad<0.001 \quad-15.4 \quad-21.9$ to $\quad<0.0001$

excess $-5.0 \quad-8.8$

$\begin{array}{lllllll}\text { TTNtv*Alcohol excess } & -9.7 & -17.5 & 0.02 & -14.4 & -21.9 & 0.0001\end{array}$ interaction§

to -1.9

to -7.0

Table shows unadjusted univariable and adjusted multivariable analyses of the effect of TTNtv and alcohol excess on baseline left ventricular ejection fraction.

*Adjusted for gender, a family history of DCM, a history of atrial fibrillation and the presence of mid wall fibrosis (late gadolinium enhancement on CMR) and NYHA class.

$\S$ i.e. the effect of TTNtv and alcohol excess compared to either TTNtv alone or alcohol excess alone. 